\title{
Application of the Monte Carlo method to analyze materials used in flat panel detectors to obtain X-ray spectra
}

\author{
Sergio Gallardo*, Fausto Pozuelo, Andrea Querol, José Ródenas and Gumersindo Verdú \\ Universitat Politècnica de València, Camí de Vera s/n, 46022 València, Spain; \\ *Corresponding Author, E-mail: sergalbe@upv.es
}

\begin{abstract}
An accurate knowledge of the photon spectra emitted by X-ray tubes in radiodiagnostic is essential to better estimate the imparted dose to patients and to improve the quality image obtained with these devices. In this work, it is proposed the use of a flat panel detector together with a PMMA wedge to estimate the actual X-ray spectrum using the Monte Carlo method and unfolding techniques. The MCNP5 code has been used to model different flat panels (based on indirect and direct methods to produce charge carriers from absorbed X-rays) and to obtain the dose curves and system response functions. Most of the actual flat panel devices use scintillator materials that present K-edge discontinuities in the mass energy-absorption coefficient, which strongly affect the response matrix. In this paper, the applicability of different flat panels for reconstructing X-ray spectra is studied. The effect of the mass energy-absorption coefficient of the scintillator material has been studied on the response matrix and consequently, in the reconstructed spectra. Different unfolding methods are tested to reconstruct the actual X-ray spectrum knowing the dose curve and the response function. It has been concluded that the regularization method Modified Truncated Singular Value Decomposition (MTSVD) is appropriate to unfold Xray spectra in all the scintillators studied.
\end{abstract}

KEYWORDS: Flat Panel, Monte Carlo, unfolding, scintillator materials.

\section{Introduction}

Obtaining X-ray spectra in radiodiagnostic units is a complex task mainly due to the high photon flux and the low energy of particles emitted by the tube. To reduce the high fluence reaching the detector, a dispersive or attenuating material is needed. The X-ray spectrum can be estimated using a flat panel detector and an attenuating material.

Flat panels are based on transforming the absorbed X-rays into charge carriers by means of direct or indirect methods. In direct devices, amorphous Selenium (a:Se) is normally used to directly transform photon fluence into current. In indirect devices, a scintillator material absorbs the X-rays and converts them into visible light photons that pass onto a photodiode array. A semiconductor foil is used to convert visible light photons into charge carriers. Normally, amorphous Silicon is preferred as a semiconductor foil to convert visible light photons into charge carriers due to its high rate for Detective Quantum Efficiency (DQE). Although silicon has outstanding electronic properties, it is not a particularly good absorber of X-ray photons. For this reason, X-rays first impinge upon scintillators usually made from either Gadolinium OxySulfide (GOS) or Cesium Iodide. Stopping power, speed and luminosity are the main characteristics to take into account to have a good scintillator material. Stopping power is maximized by maximizing density and atomic number. High density and stopping power are important for reducing the amount of the scintillator material needed (Derenzo and Weber, 2003). Alternative materials like Cadmium Telluride and Mercury Iodide are being studied as they have better stopping power (Hubbell and Seltzer, 2011). Taking into account these considerations, flat panels with different scintillator materials have been analysed.

A PMMA (polymethylmethacrylate) wedge has been used as attenuating material. This wedge is placed between the X-ray focus and the flat panel. When the flat panel is irradiated, a gray-scaled image is obtained. From this image, an absorbed dose curve can be easily obtained. The absorbed dose curve obtained using different scintillator materials can be transformed into tissue equivalent dose multiplying the dose in the first material by the ratio of mass energy absorption coefficients for the second and first material taking into account the photons of interest (always under conditions of secondary charged particle equilibrium). 
In this frame, the Monte Carlo code MCNP5 (X-5 Monte Carlo Team, 2003) has been used to simulate the energy absorption in the flat panel and to obtain the dose curve corresponding to certain working conditions of a X-ray tube. The developed MCNP5 model can be used to analyse the behaviour of different scintillators and to determine their response function depending on the X-ray energy. The response function of different materials used in direct and indirect measurements have been determined.

Simulating several monochromatic X-ray beams and calculating the dose curve for each one, the response matrix of the system can be obtained. Knowing the response matrix and the dose curve, the primary X-ray spectrum can be unfolded. However, it has been proved that response matrices characterizing the described problem are illposed.

In order to unfold the primary X-ray spectrum, regularization methods can be applied.

Unfolding methods are widely used in different fields of gamma and X-ray spectrometry, environmental radiation, imaging and nuclear data. For instance, the Maximum Entropy Method (MEM) has been successfully used for gamma ray unfolding (Los Arcos J.M, 1996) and for neutron unfolding with the code MAXED (Reginatto et al., 2002). Regarding to nuclear data, the general problem of estimating parameters in nuclear spectroscopy can be undertaken using stochastic numerical Bayesian technique and the reversible-jump Markov-chain Monte-Carlo method (Gulam Razul et al., 2003). Bayesian and Maximum Entropy methods have been used in diagnostic measurements with compact neutron spectrometers (Reginatto et al., 2008). All these methods, partially using the Monte Carlo method, represent some of the new perspectives of spectra and imaging unfolding

In this work, unfolding methods based on the Singular Value Decomposition (SVD) (Golub and Van Loan, 1996) of a response matrix have been used. Specifically, the Truncated Singular Value Decomposition (TSVD), the Modified Truncated Singular Value Decomposition (MTSVD) (Hansen et al. 1992), the Dump Singular Value Decomposition (DSVD) (Hansen et al., 1992), and the Tikhonov method (Hansen, 1999) have been used. These methods have been tested simulating the dose curve for different X-ray spectra extracted from the IPEM 78 Report (Cranley et al., 1997) and verified with experimental measurements.

\section{Methodology}

\section{The Monte Carlo model}

The Monte Carlo code MCNP5 (X-5 Monte Carlo Team, 2003) has been used to model an X-ray source, a PMMA wedge and different flat panel detectors including a:Se, CdTe CsI(Tl), GOS and HgI2.

The theoretical spectra for certain working conditions of the X-ray tube, has been established using the IPEM78 Report Catalogue (Cranley et al., 1997). This actual version uses the XCOM photon cross section library (Berger and Hubbell, 1987) to calculate linear attenuation coefficients of various materials. The unattenuated photon spectra are given for tungsten targets, tube potential from $30 \mathrm{kV}$ to $150 \mathrm{kV}$, and target angles from $6^{\circ}$ to $22^{\circ}$. The ripple value can be changed from 0 to $30 \%$. In this study a Tungsten anode tube has been considered.

The PMMA wedge is placed between the X-ray focus and the flat panel, as it can be seen in Figure 1. A scheme of a generic flat panel model it is shown in Figure 2 with all the materials that conforms a typical flat panel detector. The carbon filter is used to prevent the X-ray penetration the scintillator layer being interfered and absorbed visible light. The scintillator material absorbs the X-rays and converts them into visible light photons that pass onto a photodiode array (fibre optic). The semiconductor foil is used to convert visible light photons into charge carriers.

When the flat panel is irradiated, it registers an absorbed dose gradient due to the attenuation of X-rays produced in the wedge. For certain working conditions (high voltage, filter thickness and current) of the X-ray tube, an absorbed dose curve is obtained.

The absorbed dose curve is directly related to the primary spectrum by means of a Response function. In most applications, the Response function can be approximated by a matrix, which can be obtained using the Monte Carlo method. The photon fluence has been measured in the scintillator layer at 25 different positions along the $\mathrm{X}$ axis using a F4MESH tally (X-5 Monte Carlo Team, 2003). Photon fluence can be converted into dose using the 
mass energy-absorption coefficient $\left(\frac{\mu_{e n}}{\rho}\right)$ provided by the National Institute of Standards and Technology (NIST) (Hubbell and Seltzer, 2011). The mass energy-absorption coefficients for each material considered in the MCNP5 models are shown in Figure 3.

Common scintillators used in commercial flat panels present a K-edge in the energy range of interest, as it can be seen in Figure 2: GOS (at $50 \mathrm{keV}$ ), $\mathrm{HgI} 2$ (at 33.17 and $83.1 \mathrm{keV}$ ), CdTe (at 26.7 and $31.8 \mathrm{keV}$ ) and CsI (at 33.17 and $35.98 \mathrm{keV}$ ), all of them used in indirect methods. On the other hand, a:Se (direct method) does not present any $\mathrm{K}$-edge in the energy range studied ( 5 to $120 \mathrm{keV}$ ). Using this model as a reference, different configurations have been evaluated changing the flat panel configuration. The thickness of scintillator is different in each detector (GOS $0.14 \mathrm{~mm}$ (Hamamatsu, 2007), $\mathrm{HgI}_{2} 0.25 \mathrm{~mm}$ (Iwanczyk et al., 2001), CdTe $0.2 \mathrm{~mm}$ (Izumi et al., 2001) and CsI 0.6 mm (Chabbal et al., 2002)). In the direct flat panel the thickness of a:Se is $1 \mathrm{~mm}$ (Izumi et al., 2001).

Each model has been run simulating 50 million particles in order to ensure a relative error in F4MESH lower than $1 \%$. MODE P, E has been activated to follow tracks of photons and electrons. A default cutoff of $1 \mathrm{keV}$ for electrons has been considered.

Figure 4 shows the absorbed dose curves obtained for each case simulating different $\mathrm{X}$-ray spectra varying the high voltage $(60,70,80,90$ and $100 \mathrm{kV})$. In all simulations, it has been considered Tungsten X-ray spectra with $12^{\circ}$ anode angle and $2 \mathrm{~mm}$ thickness of Aluminium filter. All the curves represent absorbed dose in $\mu \mathrm{Gy}$ per emitted photon.

As it can be seen the efficiency of the detection produces important variations in the dose curves. Varying the scintillator material there are some bands of energy where absorbed dose curves are clearly separated, while in other intervals of energy they are overlapped.

In the case of CdTe, it can be seen that the absorbed dose corresponding to a $100 \mathrm{kV} \mathrm{X-ray} \mathrm{spectrum} \mathrm{is} \mathrm{lower} \mathrm{than}$ the corresponding to $90 \mathrm{kV}$. This fact represents an important disadvantage when primary X-ray spectrum is wanted to be determined using the absorbed dose curves. This point will be discussed in section III.

The MCNP5 model is used to determine different response matrices of the system, one per each flat panel detector. With this aim, different monochromatic beams have been simulated, calculating the absorbed dose curve for each of them. Figure 5 shows the response matrix obtained for each scintillator. In this figure it can be seen the effect of the efficiency on the absorbed dose when the X-ray energy is varied.

The K-edge has an important effect on the response function of the system. Due to the K-edge, the relation between absorbed dose and beam energy is usually not linear. For example, in energies below the GOS K-edge the maximum dose per emitted photon is found at $30 \mathrm{keV}$. Similar behavior is observed in the other scintillator materials.

Both features (K-edge and efficiency variation) produce an important effect on the reconstruction of the primary spectrum (section III).

\section{The unfolding method}

The relation between the absorbed dose curve and the primary spectrum can be defined by the Response matrix $(\mathrm{R})$, which can be obtained simulating the response of monochromatic photon beams:

$R \vec{s}-\vec{m}$

where $\vec{s}$ is the unknown primary spectrum and $\vec{m}$ is the dose curve registered. This methodology has been applied to the different scintillator materials (a-Se, GOS, CsI(Tl), CdTe and $\mathrm{HgI} 2$ ) to obtain their Response matrix.

Once $\mathrm{R}$ is known, the equation (1) theoretically permits to obtain the primary spectrum $\vec{s}$. But as the determination of this matrix is affected by some errors an approximation $\overrightarrow{\tilde{S}}$ to $\vec{S}$ is chosen in such a way that minimizes the 2 - 
norm of the residual vector $\|R \overrightarrow{\tilde{s}}-\vec{m}\|_{2}$. Singular values of $\mathrm{R}$ rapidly decay to 0 , being the last singular values very small.

This fact can be seen in Figure 6, where the singular values of different scintillator materials considered in this work are shown. These small singular values make matrix $\mathrm{R}$ to be ill conditioned. Condition number of the response matrices are 3.52 $\cdot 105$ for a-Se, 3.32 105 for CdTe, 3.13.105 for CsI, 2.99·105 for GOS and 1.76 106 for $\mathrm{HgI}_{2}$. The high value of the condition number allows pointing out that these response matrices are ill conditioned.

Due to the fact that $\mathrm{R}$ can be considered as rank deficient, there are a large number of solutions for the Least Squares problem $\|R \overrightarrow{\tilde{S}}-\vec{m}\|_{2}$. A fundamental result about rank deficient matrices, which can be derived from the Singular Values Decomposition (SVD) of $\mathrm{R}$, is that closest rank-k approximation $\mathrm{Rk}$ to $\mathrm{R}$ is obtained by truncating the SVD expansion at k, removing the parts of the solution corresponding to the smallest singular values (Golub and Van Loan, 1996). The Truncated SVD (TSVD) and the Modified TSVD (MTSVD) regularization methods are based on this observation in that one solves the problems:

$\min \|\overrightarrow{\tilde{s}}\|_{2}$ subject to $\min \left\|R_{k} \overrightarrow{\tilde{s}}-\vec{m}\right\|_{2}$

$\min \|L \overrightarrow{\tilde{s}}\|_{2}$ subject to $\min \left\|R_{k} \overrightarrow{\tilde{s}}-\vec{m}\right\|_{2}$

respectively.

In the MTSVD method, equation 3, the 2-norm $\|\overrightarrow{\tilde{s}}\|_{2}$ is replaced by the seminorm $\|L \overrightarrow{\tilde{s}}\|_{2}$, where $L$ is a discrete approximation to the p'th derivative operator (Hansen et al., 1992).

Finally, Tikhonov regularization method (Hansen, 1999) is based on a modification of the Fredholm integral equation of the first kind (a classical example of an ill-posed problem) that is intended to stabilize its solution. This is achieved by constructing the following functional:

$f(x)=\|R \overrightarrow{\widetilde{s}}-\vec{m}\|^{2}+\lambda\|L \overrightarrow{\widetilde{s}}\|$

The idea is to define the regularized solution $\overrightarrow{\widetilde{s}}$ as the minimizer of the following weighted combination of the residual norm and the side constraint

$\overrightarrow{\widetilde{s}}=\operatorname{argmin}\left\{\|R \overrightarrow{\widetilde{s}}-\overrightarrow{\mathrm{m}}\|_{2}^{2}+\lambda^{2} \| L \overrightarrow{\widetilde{s}}_{2}^{2}\right\}$

where the regularization parameter $\lambda$, controls the weight given to minimization of the side constraint relative to minimization of the residual norm. L takes different forms in accordance with the order of regularization. Different $\mathrm{L}$ operators can be used $\left(\mathrm{I}, \mathrm{L}_{1}\right.$ or $\left.\mathrm{L}_{2}\right)$. In this paper it has been chosen the second order Tikhonov regularization, $\mathrm{L}_{2}$.

$\mathbf{L}_{1}=\left[\begin{array}{cccccc}1 & -1 & & & & \\ & 1 & -1 & & & \\ & & 1 & \ddots & & \\ & & & \ddots & -1 & \\ & & & & 1 & -1 \\ & & & & & 1\end{array}\right]$ 


$$
\mathbf{L}_{2}=\left[\begin{array}{cccccc}
2 & -1 & & & & \\
-1 & 2 & -1 & & & \\
& -1 & 2 & \ddots & & \\
& & \ddots & \ddots & & \\
& & & & 2 & -1 \\
& & & & -1 & 2
\end{array}\right]
$$

A less known regularization method, which is based on the SVD or the GSVD is the damped SVD/GSVD. Here, instead of using filter factors 0 and 1 as in TSVD one introduces a smoother cut-off by means of filter factors $f_{i}$ defined as

$$
f_{i}=\frac{\sigma_{i}}{\sigma_{i}+\lambda \mu_{i}}
$$

for $\mathrm{L} \neq I_{n}$

These filter factors decay slower than the Tikhonov filter factors and thus, introduce less filtering (Hansen et al., 1992).

The quality of the unfolding method results strongly depends on the regularization parameter ( $\mathrm{k}$ and $\lambda$ ). If it is too small, the result will show artificial peaks. If it is too large, the result will be over-smoothed. For this reason, a reliable method for determination of the regularization parameter is essential to solve the inverse problem.

Some methods are based on tracking the changes in the size of the regularized solution versus the size of the corresponding residual to determine an optimal value of $\mathrm{k}$ and $\lambda$. The procedure represented in graphical form is referred to as the L-curve criterion (Hansen, 1994, 1998). The use of such a criterion in connection with ill-posed Least Squares problems goes back to Miller (1970), and Lawson and Hanson (1974). The L-curve criterion is clearly illustrated and extensively applied to the analysis of discrete ill-posed problems by Hansen (1992). More properties of the L-curve are derived by Hansen and OLeary (1993) where it was also shown that the characteristic L-shaped corner is better pronounced in a double logarithmic plot. L-curve consists of a relatively vertical segment and a relatively horizontal line in an ideal setting. In this curve, the 2-norm of the solution vector $\|x\|_{2}$ or $\|L x\|_{2}$ is plotted versus the 2-norm of the residual vector $\|A x-b\|_{2}$ for different values of the $\mathrm{k}$ parameter. Some authors recommend finding the truncation parameter $\mathrm{k}$ that is closest to the maximum curvature point, that is, selecting the value corresponding to the L-shaped corner.

Figure 7 shows the L-curves obtained applying TSVD, MTSVD and Tikhonov methods with the response matrix corresponding to a:Se.

Other method for choosing a good regularization parameter is the Discrete Picard Condition (DPC), recognized by Varah (1979) and analyzed by Hansen $(1988,1990)$. The right-hand side vector b of the Least Squares problem $\|A x-b\|$ satisfies the DPC if the Fourier coefficients $\left|u_{i}^{T} b\right|$ decay, on the average, to zero faster than the singular values $\sigma$ i. Figure 8 shows the Picard condition for each scintillator material applied to MTSVD method.

Let $\gamma_{i}=u_{i}^{T} b / \sigma_{i}$. Then on the average, $\left|\gamma_{i}\right|$ decreases until some point $\gamma_{P}$, where it starts to increase. Write $\mathrm{b}$ as $\mathrm{b}$ $=$ bexact + berror, where bexact is the exact right-hand side vector for the problem and berror is a perturbation vector. Define $\gamma_{i}^{\text {exact }}=u_{i}^{T} b^{\text {exact }} / \sigma_{i}$ and $\gamma_{i}^{\text {error }}=u_{i}^{T} b^{\text {error }} / \sigma_{i}$. Then $\gamma_{i}=\gamma_{i}^{\text {exact }}+\gamma_{i}^{\text {error }}$. Additionally, it is assumed that, on the average, $\left|\gamma_{i}^{\text {exact }}\right|$ decreases toward zero and $\left|\gamma_{i}{ }^{\text {error }}\right|$ increases from zero. That is, the exact problem $A_{x}=\mathrm{b}^{\text {exact }}$ satisfies the DPC completely and the error problem $A_{x}=\mathrm{b}^{\text {error }}$ does not satisfy the DPC at all. With these assumptions, it can be seen that for the decreasing part of the $\gamma_{i}, \gamma_{i}^{\text {exact }}$ is the dominant component; and $\gamma_{i}^{\text {error }}$ is the dominant one for the increasing part.

Taking into account all these considerations, regularization parameters $\mathrm{k}$ and $\lambda$ can be objectively determined. In Table 1 it is listed the regularization parameters $\mathrm{k}$ and $\lambda$ obtained applying the L-curve criterion to each response 
matrix and dose curve for each unfolding method. As it can be seen, the optimal value of the truncation parameter is almost constant in the voltage interval considered for each unfolding method. For TSVD method $\mathrm{k}=4$ has been used in all the cases except for the GOS $(\mathrm{k}=5)$. For MTSVD has been considered $\mathrm{k}=3$ in all the cases. More differences between each material are found in the Tikhonov method $(\lambda$ a:Se $=8.50$ e- $08, \lambda \mathrm{CdTe}=4.40$ e- $07, \lambda$ CsI, GOS, $\left.\mathrm{HgI}_{2}=2.40 \mathrm{e}-07\right)$.

\section{Results and Discussion}

The optimal choice of the truncation parameter, $\mathrm{k}$ or $\lambda$, contributes to obtain a best fit of unfolded spectrum respect to the theoretical primary beam spectrum. When $\mathrm{k}$ or $\lambda$ is greater than the value given by the L-curve corner, fluctuations and noise increase in the unfolded spectrum. On the other hand, when $\mathrm{k}$ or $\lambda$ is lower, unfolded spectrum is over smoothed, loosing information.

In Figure 9, it is shown a comparison between theoretical $100 \mathrm{kV}$ spectrum and unfolded spectra obtained applying the MTSVD method for different regularization parameter $\mathrm{k}$. As it has been said, solution is highly affected by the choice of $\mathrm{k}$. Furthermore, the characteristic lines of tungsten are not present in the unfolded spectra. An analogous analysis can be done in the case of Tikhonov unfolding.

Once the k parameter has been fixed for each unfolding method and each response matrix, the methodology has been tested for different tube working conditions varying the peak high voltage.

Figures 10 to 16 show comparisons between unfolded and IPEM78 theoretical spectra for $70 \mathrm{kV}$ and different flat panel configurations. TSVD, MTSVD, DSVD and Tikhonov methods have been applied to reconstruct primary spectra.

In the case of a:Se, MTSVD $(\mathrm{k}=3)$ successfully fits theoretical spectra. Figure 10 shows a comparison of unfolded spectra (using the regularization methods MTSVD and Tikhonov). In all cases, a smooth solution is obtained thanks to the derivative operator $\mathrm{L}_{2}$. Bremsstrahlung distribution obtained with MSTVD adequately reproduces the theoretical spectra. However, the maximum of the Bremsstrahlung continuous is reached $10 \mathrm{keV}$ above the maximum of the theoretical spectra. The most important discrepancies are found in the energy range defined between 40 and $70 \mathrm{keV}$. Regarding to Tikhonov regularization $(\lambda=8.50 \mathrm{E}-08)$, smooth spectra are obtained, but Bremsstrahlung continuous is shifted about $10 \mathrm{keV}$ to the low energy range.

When $\mathrm{L}_{2}$ operator is not considered, important discrepancies appear (fluctuations and distortions of the spectra). This effect can be observed in Figure 11. In this case, TSVD, DSVD and Tikhonov (L=I) have been applied to unfold spectra of 70 and $100 \mathrm{kV}$. Reconstruction obtained with TSVD method shows slight fluctuations in the whole energy interval. TSVD unfolded spectrum present more important discrepancies when high voltage is reduced. In fact, only an accurate spectrum is obtained for $100 \mathrm{kV}$. DSVD method does not offer acceptable results in any case.

In general, using TSVD, DSVD and Tikhonov ( $\mathrm{L}=\mathrm{I})$ important fluctuations and noise appear in unfolded spectra. Furthermore, solution is distorted and shifted to the high energy range. In the case of DSVD and Tikhonov $(\mathrm{L}=\mathrm{I})$, the regularization methods are not able to adequately determine the maximum energy of the Bremstrahlung continuous. This fact is more evident in the $70 \mathrm{kV}$ spectrum (in both methods, the maximum energy of the distribution reaches $100 \mathrm{keV}$ ). In any case, characteristic lines are not reproduced.

As a conclusion, it can be said that the derivative operator, $\mathrm{L}_{2}$, is necessary to smooth the spectra in order to obtain an acceptable estimation of the primary spectra.

Figure 12 shows the unfolded spectra obtained when CdTe response matrix is considered. It can be observed the same general trends explained for a:Se. It is needed the derivative operator $\mathrm{L}_{2}$ to obtain a smooth and adequate solution. The weak point of choosing CdTe to reconstruct spectra is its poor behaviour for voltages greater than 90 $\mathrm{kV}$. In Figure 12 it can be clearly seen that the worse reconstructions are found for $100 \mathrm{kV}$, independently of the unfold method used. This effect can be attributed to the reduction of detection efficiency for energies greater than 
$90 \mathrm{keV}$. Unfolded spectra obtained with TSVD and Tikhonov ( $\mathrm{L}=\mathrm{I})$ do not fit theoretical spectra for any tested voltage (70 up to $100 \mathrm{kV}$ ), as it can be seen in Figure 13. It is the same behaviour than with a:Se.

In Figures 14, 15 and 16, it is shown the unfolded spectra obtained applying the MTSVD and Tikhonov $\left(\mathrm{L}_{2}\right)$ regularization methods to CsI, GOS and $\mathrm{HgI} 2$ matrices, respectively. The behaviour of solutions is quite similar in all cases. MTSVD unfolded spectra are shifted to the low energy range, and Tikhonov spectra to high energies. Kedge appearing at different energies do not produce any particular effect on the reconstructed spectra. It can be stated that K-edge of scintillator does not have a relative importance to cause the ill-posed problem.

From all these results, some points should be highlighted: K-edge of different response matrices do not alter the ill-posed condition of the problem and do not affect the quality of the unfolded spectrum. The condition number of the response matrix does not depend on K-edge. It has been proved that the response matrix of all material analysed have a similar condition number (about 105), also with similar singular value decomposition. However, the efficiency of some scintillators is gradually reduced for energies up to $100 \mathrm{keV}$, for example CdTe. In this case, regularization methods such as MSTVD or Tikhonov do not adequately unfold spectra. On the other hand, it is necessary to consider a derivative operator (L2) to obtain a smooth unfolded spectrum. In general trends, from the mathematical point of view, it seems that the scintillator material does not represent a relevant aspect to unfold primary spectra. However, choosing the adequate regularization method is very important to obtain acceptable unfolded primary spectra.

\section{Conclusions}

The applicability of the system PMMA wedge-Flat panel to reconstruct primary X-ray spectra has been analysed. It has been stated the suitability of the MTSVD and Tikhonov regularization methods to unfold X-ray spectra in the considered energy range.

A MCNP5 model to simulate the actual acquisition process has been developed. Different materials used in commercial flat panels have been tested using this model. The response matrix corresponding to each scintillator has been obtained simulating several monoenergetic photon beams. The response matrices obtained have a large condition number, pointing out an ill-posed problem. Two features can, a priori, affect the quality of the solution: variation of efficiency depending on energy and the K-edge of the scintillator. To study these hypotheses, five response matrices of different scintillators have been analysed and used to unfold primary X-ray spectra in the radiodiagnostic range.

Regularization methods such as MTSVD and Tikhonov are able to unfold primary spectra when a derivative operator is used to smooth the solution. TSVD, DSVD and Tikhonov (using L as identity) do not offer good results in any case. In any case, characteristic lines are not properly unfolded.

The K-edge does not have a relevant effect in the unfolded spectra. However, the variation of efficiency strongly affects the quality of the unfolded spectrum.

From the point of view of X-ray unfolding, it has been proved that CdTe does not allow obtaining acceptable results for voltages greater than $90 \mathrm{kV}$.

It is recommended the use of the regularization MTSVD method for any kind of scintillator used.

The effect of the K-edge is negligible during the unfolding process.

\section{References}

Berger, M. J., Hubbell, J. H., 1987. XCOM: photon cross sections on a personal computer, NBSIR 87-3597, National Bureau of Standards (US). 
Chabbal, J., Arques, M., Peyret, O., 2002. Flat panel X-ray detector, Troisième Conférence Internationale sur les Nouveaux développements en photodetection, Beaune, France.

Cranley, K., Gilmore, B. J., Fogarty, G. W. A., Deponds L., 1997. Catalogue of diagnostic X-ray spectra and other data, IPEM Report No. 78, The Institute of Physics and Engineering in Medicine, York.

Derenzo, S. E., Weber, M. J., Bourret-Courchesne E., Klintenberg M. K., 2003. The quest for the ideal inorganic scintillator", Nucl. Instrum. Methods, 505, 111-117.

Golub, G. H., Van Loan, C.F., 1996. Matrix Computations. Third ed. The Johns Hopkins University Press.

Gulam Razul S., Fitzgerald W.J., Andrieu C., Bayesian model selection and parameter estimation of nuclear emission spectra using RJMCMC, 2003. Nuclear Instruments and Methods in Physics Research Section A: Accelerators, Spectrometers, Detectors and Associated Equipment, 497(2-3), 492-510.

Hamamatsu comercial catalogue, 2007. www.hamamatsu.com.

Hansen, P.C., 1988. Computation of the Singular Value Expansion, Computing, 40, 185-199.

Hansen, P.C., 1990. The Discrete Picard Condition for Discrete Ill-posed Problems, BIT, 30, 658-672.

Hansen, P.C., 1992. Analysis of discrete ill-posed problems by means of the L-curve, SIAM Rev. 34, 561-580.

Hansen, P.C., Sekii, T., Shibabhashi, H., 1992. The modified truncated SVD method for regularization in general form", SIAM J. Sci. Comput., 13, 1142-1150.

Hansen, P.C., Oleary, D.P., 1993. The use of the L-curve in the regularization of discrete ill-posed problems, SIAM J. Sci. Comput. 14, 1487-1503.

Hansen, P.C., 1994. Numerical Algorithms, 6, 1-35.

Hansen, P.C., 1998. Rank-Deficient and Discrete Ill-Posed Problems, SIAM, Philadelpia.

Hansen, P.C., 1999. Regularization tools Version 3.0 for Matlab 5.2, Numer. Algorithms, 20, 195-196.

Hubbell, J. H., Seltzer, S. M., Last updated 2011. Tables of X-Ray Mass Attenuation Coefficients and Mass Energy-Absorption Coefficients from $1 \mathrm{keV}$ to $20 \mathrm{MeV}$ for Elements $\mathrm{Z}=1$ to 92 and 48 Additional Substances of Dosimetric

Interest, National Institute of Standards and Technology. www.nist.gov.

Iwanczyk, J.S., Patt, B.E., Tull, C.R., MacDonald, L.R., Skinner, N., Hoffman, E.J., Fornaro, L., Mussio, L., Saucedo, E., Gancharov, A., 2001. Mercuric Iodide Polycrystalline Films, P Soc. Photo-opt. Ins. III, 4508, 28-40.

Izumi, Y., Teranuma, O., Sato, T., Uehara, K., Okada, H., Tokuda, S., Sato, T., 2001. Development of Flat-Panel $\mathrm{X}$-ray Image Sensors.

Lawson, C. L., Hanson, R. J., 1974. Solving Least Squares Problems, Prentice-Hall, Englewood Cliffs.

Los Arcos J.M, Gamma-ray spectra deconvolution by maximum-entropy methods, 1996. Nuclear Instruments and Methods in Physics Research. Section A, Accelerators, Spectrometers, Detectors and Associated Equipment, 369(2-3), 634-636.

Miller, K., 1970. Least squares methods for ill-posed problems with a prescribed bound, SIAM J. Math. Anal. 1, 52-74. 
Reginatto M, Goldhagen P, Neumann S, Spectrum unfolding, sensitivity analysis and propagation of uncertainties with the maximum entropy deconvolution code MAXED, 2002. Nuclear Instruments and Methods in Physics Research Section A: Accelerators, Spectrometers, Detectors and Associated Equipment, 476, 242-246.

Reginatto M, Zimbal A, 2008. Bayesian and maximum entropy methods for fusion diagnostic measurements with compact neutron spectrometers. Rev Sci Instrum, 79.

Varah, J. M., 1979. A Practical Examination of Some Numerical Methods for Linear Discrete Ill-posed Problems, SIAM Rev., 21, 100-111.

X-5 MONTE CARLO TEAM, 2003. MCNP - A General Monte Carlo N particle Transport Code, Version 5 LAUR-03-1987, Los Alamos National Laboratory. 
Figure 1: Geometry layout of the system

Figure 2: Flat panel materials

Figure 3: Mass Energy Absorption Coefficient $\left(\mathrm{cm}^{2} / \mathrm{g}\right)$ NIST

Figure 4: Absorbed Dose curves for each material

Figure 5: Response matrices

Figure 6: Singular values

Figure 7: L-curve criterion (a:Se). TSVD, MTSVD and Tikhonov

Figure 8: Picard condition

Figure 9: MTSVD unfolding with different $k$ values

Figure 10: Unfolded spectra a:Se. MTSVD and Tikhonov

Figure 11: Unfolded spectra a:Se. TSVD, DSVD and Tikhonov (L=I)

Figure 12: Unfolded spectra CdTe. MTSVD and Tikhonov

Figure 13: Unfolded spectra CdTe. TSVD and Tikhonov (L=I)

Figure 14: Unfolded spectra CsI. MTSVD and Tikhonov

Figure 15: Unfolded spectra GOS. MTSVD and Tikhonov

Figure 16: Unfolded spectra HgI2. MTSVD and Tikhonov

Table 1: Regularization parameters 